EXTENDED REPORT

\title{
Impact of simulated light scatter on scanning laser Doppler flowmetry
}

\author{
S T Venkataraman, C Hudson, E Harvey, J G Flanagan
}

Br J Ophthalmol 2005;89:1192-1195. doi: 10.1136/bjo.2004.065169

See end of article for authors' affiliations ......................

Correspondence to: Dr C Hudson, School of Optometry, University of Waterloo, Waterloo, Ontario, N2L 3G1,

Canada; chudson@ scimail.uwaterloo.ca

Accepted 1 April 2005
Aim: To determine the impact of simulated light scatter on scanning laser Doppler flowmetry (SLDF) assessment of retinal capillary blood flow and retinal image quality.

Methods: One eye of 10 normal subjects (mean (SD) age 24 (1.7) years, range 22-27) was randomly selected. Varying concentrations of polystyrene microspheres were suspended in optically clear cells to simulate light scatter. The microsphere concentrations used were $0.05 \%, 0.03 \%, 0.02 \%, 0.01 \%$, and a cell containing only water. LogMAR visual acuity and contrast sensitivity were measured both with and without cells. Optimal focus and alignment was established by acquiring three SLDF images each of the optic nerve head $(\mathrm{ONH})$ and of the macula using the Heidelberg retina flowmeter (HRF) with no cell in place. SLDF images were subsequently acquired with each of the light scatter cells mounted in front of the HRF. The group mean retinal capillary blood flow was compared using repeated measures analysis of variance (reANOVA) as a function of microsphere concentration.

Results: Retinal capillary blood flow increased significantly in the $\mathrm{ONH}$, nasal macula, fovea, and temporal macula with increasing microsphere concentration $(p<0.0001)$. Using Dunnett's post hoc test, retinal capillary blood flow was found to be significantly increased relative to the no cell condition for the $0.03 \%$ and $0.05 \%$ cell concentrations.

Conclusions: Simulated light scatter produces an artifactual increase in retinal capillary blood flow. The impact of cataract on SLDF measurements has yet to be determined.
C ataract results in intraocular light scatter due to change in refractive index and opacification of the crystalline lens leading to degradation of retinal image quality. ${ }^{12}$ In humans, the aggregation of lens crystallins forms high molecular weight (HMW) aggregates of 300-500 nm diameter resulting in cataract. ${ }^{3}$ Degradation of retinal image quality and contrast in a cataractous eye is primarily caused by forward light scatter. ${ }^{5}$

Non-invasive digital imaging techniques designed to improve the detection and clinical management of retinal disease are now used commonly. Assessment of the influence of ocular media opacities on the outcome of these new imaging techniques is important because cataract frequently occurs concomitantly with retinal disease. A number of reports have studied the influence of simulated light scatter on visual field performance and have shown a decrease in visual field sensitivity. ${ }^{6-8}$ We have recently developed a simulated light scatter model and have demonstrated its impact on fundus visualisation using a digital retinal camera. ${ }^{9}$

The purpose of the study was to determine the influence of simulated light scatter on scanning laser Doppler flowmetry (SLDF) assessment of retinal capillary blood flow and retinal image quality.

\section{METHODS \\ Sample}

The study protocol was approved by the University of Waterloo, Office of Research Ethics. Ten subjects of mean (SD) age 24 (1.7) years (range 22-27) participated in the study. All participants had a LogMAR visual acuity of 0.0 or better. Subjects with the following Lens Opacity Classification System (LOCS) III $^{10}$ grading were excluded from the study: nuclear colour $>\mathrm{NC} 2$, nuclear opalescence $>\mathrm{NO} 2$; cortical $>\mathrm{C} 2$; and posterior subcapsular $\geqslant \mathrm{P} 1$.

\section{Scanning laser Doppler flowmetry (SLDF)}

The light reflected by moving red blood cells in the retina undergoes a frequency shift while light reflected from surrounding tissue remains unchanged. Using SLDF, the intensity of back scattered laser light from the retina is measured as a function of time (to produce an intensity-time curve) for each pixel within the image. The two coherent components of light interfere, resulting in an oscillation or "beat" of the measured light intensity. The frequency of the intensity oscillation is equal to the Doppler frequency shift. Fast Fourier transformation of the intensity-time curve generates a power spectrum of the Doppler shift to derive parameters of blood flow, volume, and velocity at each pixel within the image. ${ }^{11}$

SLDF measurements were undertaken using the Heidelberg retina flowmeter (HRF Version 1.03W; Heidelberg Engineering $\mathrm{GmbH}$, Dossenheim, Germany). The HRF uses a $780 \mathrm{~nm}$ wavelength diode laser to measure the intensity and thereby calculate the Doppler shift of back scattered light. The instrument has a spatial resolution of approximately $10 \mu \mathrm{m}$ and a depth resolution of 300$400 \mu \mathrm{m} .{ }^{12-14}$ The laser scanning system of the HRF enables the measurement of a $10^{\circ}$ horizontal $\times 2.5^{\circ}$ vertical field (that is, approximately $2.7 \mathrm{~mm} \times 0.7 \mathrm{~mm}$ ) with a resolution of 256 pixels $\times 64$ lines. Each line of 256 pixels is scanned 128 times at a repetition rate of $4000 \mathrm{~Hz}$. The resulting image acquisition time is 2.048 seconds.

\section{Simulated light scatter model}

Light scatter was induced using various concentrations of $500 \mathrm{~nm}$ diameter polystyrene microspheres (Polybead; Polysciences Inc, USA) suspended in sterile water. The

Abbreviations: AFFPIA, automated full field perfusion image analysis: HRF, Heidelberg retina flowmeter; ONH, optic nerve head; SLDF, scanning laser Doppler flowmetry 
diameter of the microspheres was chosen to be similar to the diameter of HMW aggregates in a cataractous lens. ${ }^{458}$ Microsphere concentrations of $0.05 \%, 0.03 \%, 0.02 \%$, and $0.01 \%$ and water only were prepared under sterile conditions (surfactant poloxamer 407 was included to prevent coagulation). ${ }^{15}$ The stability of the light scatter model was determined by assessing HRF measurements against a plain white paper background for the $0.05 \%$ cell which had not been disturbed for 24 hours, and then after vigorous shaking. The mean $(\mathrm{SD})$ value (three repeats) for $10 \times 10$ pixels was 47735 (373) arbitrary units (au), and immediately after shaking it was not significantly different (47 335 (431) au).

The various microsphere concentrations were injected into cells constructed from two $35 \mathrm{~mm}$ removable CR39 plano lenses mounted on opposite ends of a plastic ring (inner diameter $25 \mathrm{~mm}$ ), with a spacing of $4.5 \mathrm{~mm}$ between the lenses and a thickness of $2.04 \mathrm{~mm}$ each. The total volume of each cell was $2.2 \mathrm{ml}$. The cells were mounted on the objective of the HRF using a custom made adaptor that incorporated a $20^{\circ}$ tilt to minimise surface reflections (fig l).

\section{Procedures}

All participants underwent a preliminary eye examination followed by dilation of the study eye with a single drop of $0.5 \%$ tropicamide. LOCS III grading was undertaken after dilation. Three SLDF images of the optic nerve head $(\mathrm{ONH})$ and three images of the macula were acquired with no cell in place in order to establish optimal focus settings and image alignment. Three SLDF images of both the ONH and of the macula were subsequently acquired with the various cells in place. The order of cells and the measurement site were randomised between subjects and a constant focus setting and alignment was maintained based on the findings with no cells. The eye to camera distance was kept constant during image acquisition.

Fundus photographs of the study eye of one subject were acquired for all conditions using a digital fundus camera (Canon CR6-45NM, Canon, Lake Success, New York, USA) to illustrate the clinical relevance of the simulated light scatter model.

\section{Analysis of data}

Automated full field perfusion image analysis (AFFPIA) software version 3.3 and HRF custom analysis were used to generate retinal capillary blood flow values. AFFPIA excludes erroneous blood flow values caused by saccades and major blood vessels. ${ }^{16}$ The AFFPIA software was used to delineate the temporal neuroretinal rim of the ONH. In addition, a $2.5^{\circ}$ circle was centred to delineate the foveal macular area. All points temporal to the circle delineated the temporal macular area while those nasal to the circle delineated the nasal macular area. Capillary blood flow values were derived for the temporal neuroretinal rim and the various macular areas.

A custom analysis was developed partly because of the limitation of the AFFPIA software to generate blood flow values with the higher microsphere concentrations. The HRF custom analysis preserved sample size, especially with the

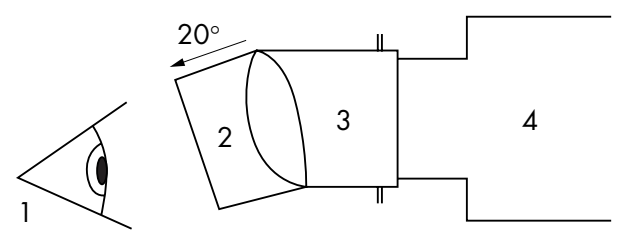

Figure 1 Schematic representation of the light scatter cell mounted in front of the objective of the Heidelberg retina flowmeter (HRF) using a custom designed adaptor to produce a standard $20^{\circ}$ tilt. 1, Subject's eye; 2 , light scatter cell; 3 , custom made adaptor used to tilt the cell by $20^{\circ} ; 4$, Heidelberg retina flowmeter. high concentration light scatter cells. HRF custom analysis consisted of a $10 \times 10$ pixel measurement window that was placed within the areas of interest. Care was taken to place the $10 \times 10$ pixel window at the same location for all images of a given subject and also within the same area that was used for AFFPIA using a transparent sheet to mark the relevant land marks and the AFFPIA area. Capillary blood flow for each pixel of the area of interest was obtained together with the brightness index of the image (DC value). A total of 100 flow values (with corresponding DC) were produced for each area analysed. Flow values with a DC range of 70-200 au were included in the analysis since this range is within the acceptable level of retinal image illumination. ${ }^{17-19}$ The data were then sorted in ascending order and any artifactual zero flow values were excluded, together with an equal number of the highest flow values. Only images with at least 35 flow values were included.

Repeated measures analysis of variance (reANOVA) of the retinal capillary blood flow values for all microsphere concentrations and all measurement regions were performed for both AFFPIA and HRF custom analyses using SAS (Version 8.02). Since AFFPIA failed to generate blood flow values in most of the subjects for the $0.05 \%$ microsphere concentration, it was not included in the statistical analysis. It was assumed that there was minimal light scatter and degradation of retinal image quality without any cell in place. Dunnett's post hoc test was performed to determine the microsphere concentration that resulted in a significant change. The DC values obtained using the HRF custom analysis for the various microsphere concentrations were also analysed in an identical manner. Pearson's correlation procedure was performed to determine whether there was a significant linear relationship between blood flow values with no cell and with increasing microsphere concentrations in all subjects.

\section{RESULTS}

The group mean baseline capillary blood flow values with the AFFPIA and HRF analysis of the ONH, nasal macula, fovea, and temporal macula are shown in table 1 .

The reANOVA for the AFFPIA showed a significant increase in capillary blood flow with increasing microsphere concentrations for the ONH $(p<0.0001)$, nasal macula $(p=0.0003)$, fovea $(p=0.0004)$, and temporal macula $(p=0.0003)$. The reANOVA for the HRF analysis also showed a significant increase in capillary blood flow with increasing microsphere concentrations for the $\mathrm{ONH}(\mathrm{p}<0.0001)$, nasal macula $(\mathrm{p}<0.0001)$, fovea $(\mathrm{p}<0.0001)$, and temporal macula $(\mathrm{p}<0.0001)$.

Dunnett's post hoc analysis of the AFFPIA data revealed a significant increase in capillary blood flow with the $0.03 \%$ cell concentration, while HRF custom analysis showed a significant increase with both $0.03 \%$ and $0.05 \%$ cell concentrations for the $\mathrm{ONH}$, nasal macula, fovea, and temporal macula relative to baseline. Capillary blood flow values of the $\mathrm{ONH}$, nasal macula, fovea, and temporal macula extracted using the AFFPIA and HRF custom analysis followed a similar trend for all light scatter cell conditions (fig 2A-D).

The reANOVA for the DC values obtained using the HRF custom analysis for all the measured regions showed a significant difference across the various microsphere concentrations $(p=0.0001$, fig 3$)$. Dunnett's post hoc analysis of the DC values revealed a significant decrease in DC values with the $0.05 \%$ cell concentration for the $\mathrm{ONH}$, nasal macula, fovea, and temporal macula relative to baseline. Pearson's correlation procedure did not show any significant linear association between blood flow values with no cells or with increasing concentrations of microspheres. There was no consistent offset observed in the effect produced by the increasing microsphere concentration. 
Table 1 Group mean (SD) baseline capillary blood flow values of the $\mathrm{ONH}$, nasal macula, fovea, and temporal macula using the AFFPIA and HRF custom analysis

\begin{tabular}{lllll}
\hline Analyses & ONH & Nasal macula & Fovea & Temporal macula \\
\hline AFFPIA & $228.86(62.84)$ & $151.96(30.30)$ & $120.25(26.08)$ & $155.88(47.90)$ \\
HRF custom & $226.68(191.63)$ & $176.52(39.46)$ & $192.65(53.92)$ & $163.89(83.11)$ \\
\hline
\end{tabular}

With no light scatter cell in place and with the cell containing only water, optimal fundus visualisation was achieved. Cells containing microsphere concentrations of $0.01 \%$ and $0.02 \%$ allowed a blurred outline of the ONH to be visible (fig 4). With higher microsphere concentrations, visualisation of fundus details was increasingly difficult.

The results of one subject were excluded because of excessive eye movement and poor image quality. AFFPIA failed to generate capillary blood flow values for $\leqslant 5$ and 9 subjects with the $0.03 \%$ and $0.05 \%$ microsphere concentrations, respectively. HRF custom analysis resulted in incomplete sets of data only for the ONH measurement site (due to our requirement of a minimum 35 flow values per pixel window) in two subjects.

\section{DISCUSSION}

To our knowledge, this is the first study to investigate the effect of artificial light scatter on retinal capillary blood flow and image quality using the HRF. AFFPIA and HRF custom analyses both showed a significant artifactual increase in capillary blood flow with increasing light scatter. Post hoc analysis of the AFFPIA data revealed a significant increase in capillary blood flow with the $0.03 \%$ cell concentration, while the HRF custom data showed a significant increase with both $0.03 \%$ and $0.05 \%$ cell concentrations for all the measured regions relative to baseline. There was a significant decrease in the DC values with the $0.05 \%$ microsphere concentration relative to baseline. The cell with water only was included as one of the conditions because it would induce some degree of image degradation and light scatter; however, the analysis showed that there was no significant difference between the no cell condition and the cell with water only. Although the

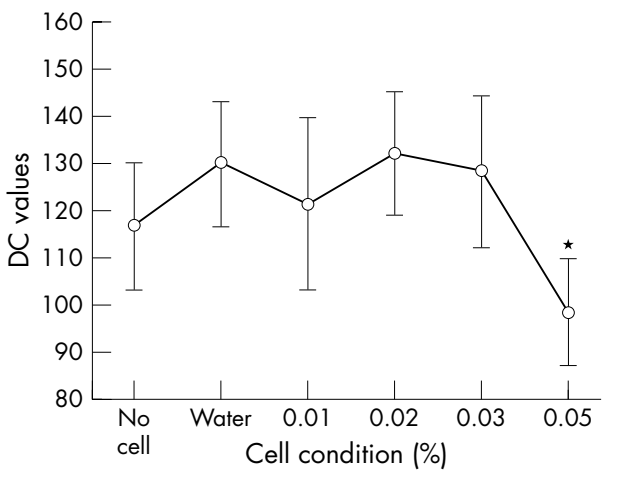

Figure 3 Group mean DC values (indicating the brightness index of the image) for all the measured regions with no cell, water only, and $0.01 \%$, $0.02 \%, 0.03 \%, 0.05 \%$ microsphere concentrations. Vertical bars denote $95 \%$ confidence intervals ( $\left.{ }^{*} p<0.05\right)$.

increase in capillary blood flow with lower concentrations of light scatter microspheres did not reach statistical significance, it might have clinical implications.

The HRF is sensitive to changes in photodetector settings. Changes in image brightness have already been shown to impact on SLDF derived estimates of retinal capillary blood flow. ${ }^{13}{ }^{18-20}$ In the current study, an increase in light scatter decreased the image brightness in all subjects leading to an increase in the HRF gain setting by the operator to optimise the view of various retinal structures, as would occur in a clinical situation. This produced a significant decrease in DC values with the highest microsphere concentration, resulting in a
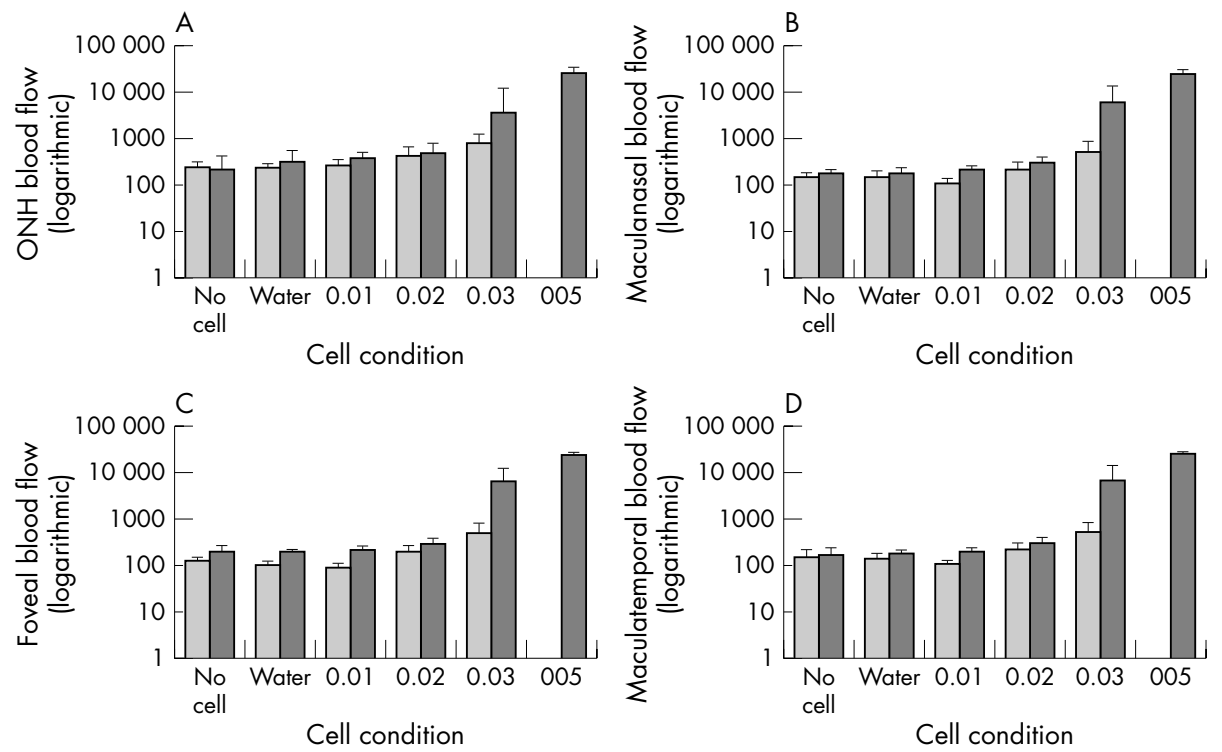

Figure 2 Group mean (SD) capillary blood flow with no cell, water only, 0.01\%, 0.02\%, 0.03\%, and 0.05\% microsphere concentrations using SLDF (light shaded bars) and HRF custom analysis (dark shaded bars) in (A) the optic nerve head (ONH), (B) the nasal macula, (C) the fovea, and (D) the temporal macula. 

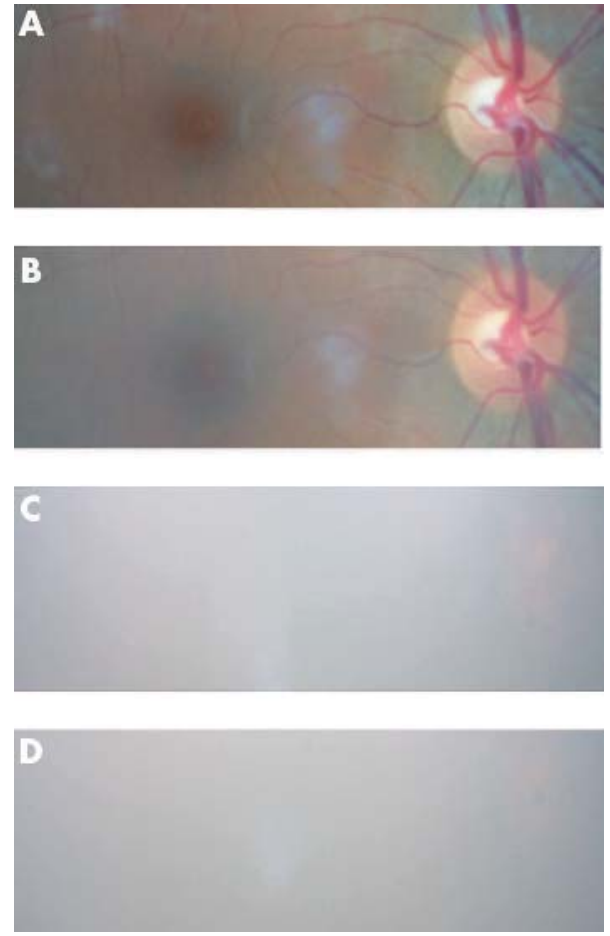

Figure 4 Fundus image of a normal subject acquired with various cell microsphere concentrations placed in front of a digital fundus camera. (A) No cell. (B) Cell with water only. (C) Cell with microsphere concentration of $0.01 \%$. (D) Cell with microsphere concentration of $0.02 \%$.

significant increase in blood flow values. DC related errors in HRF measurements have been observed in both in vitro models and in images of the human fundus. Tsang and co-workers ${ }^{20}$ suggested that darker backgrounds resulted in higher blood velocity values in an in vitro blood flow model using the HRF. An increase in retinal capillary blood flow as a result of DC changes has already been demonstrated in normal subjects using the HRF. ${ }^{19}$ However, it should be noted that both the AFFPIA and our custom HRF analyses attempted to eliminate unacceptable DC values from the raw HRF measurements.

A light scatter induced shift was observed in the $y$ axis of the power spectrum with $0.03 \%$ and $0.05 \%$ concentrations of artificial microspheres. Careful examination of the SLDF power spectrum is required to eliminate images with DC shifts beyond the valid measurement range of the HRF. The HRF has a high zero offset resulting from random variation of backscattered light and internal noise leading to changes in flow values. ${ }^{12}$ In this study, an increase in simulated light scatter exaggerated the high zero offset. This explains the significant artifactual increase in capillary blood flow with increasing light scatter.

AFFPIA resulted in exclusion of the majority of subjects as it failed to generate blood flow values for higher light scatter cell concentrations due to invalid zero offset. On the other hand, our HRF custom analysis provided capillary blood flow values for nearly all possible cell conditions with the operator defined acceptable range of DC values for all images. This feature of HRF custom analysis produced apparently valid capillary blood flow values even for the higher light scatter cell concentrations.

Thus, simulated light scatter produced an artifactual increase in capillary blood flow in the $\mathrm{ONH}$ and in various macular regions of the retina. Artificial light scatter resulted in a shift in the $y$ axis of the SLDF power spectrum. Both AFFPIA and our HRF custom analysis produced remarkably similar capillary blood flow values. We consider that SLDF images need to be analysed with caution to exclude unacceptable DC values and power spectrum shifts to derive accurate capillary blood flow values. The impact of cataract on SLDF measurements has yet to be determined; this work is currently in progress in our laboratory.

\section{ACKNOWLEDGEMENTS}

The authors thank Robin Jones for his technical expertise in the manufacture of light scatter cells, and Drs Oliver Arend and Larry Kagemann for their expert and constructive reviews of the manuscript.

\section{Authors' affiliations}

S T Venkataraman, C Hudson, J G Flanagan, Multi-Disciplinary Laboratory for the Research of Sight-Threatening Diabetic Retinopathy, School of Optometry, University of Waterloo, Waterloo, Ontario,

Canada

C Hudson, J G Flanagan, Department of Ophthalmology and Vision Science, University of Toronto, Ontario, Canada

E Harvey, Department of Statistics and Actuarial Science, University of Waterloo, Waterloo, Ontario, Canada

This work was funded by the Canadian Institutes of Health Research and by a Premier's Research Excellence Award (recipient CH).

Competing interests: none declared

\section{REFERENCES}

1 Bettelheim FA. Physical basis of lens transparency. In: Maisel H, eds. The ocular lens: structure, function and pathology. New York: Marcel Dekker, 1985:265-300.

2 Benedek GB. Theory of the transparency of the eye. Appl Optics 1971;10:459-73.

3 Bettelheim FA, Siew E. Biological and physical basis of lens transparency. In: McDevitt, eds. Cell biology of the eye. New York: Academic Press, 1982:243-97.

4 Dengler-Harles $M$, Wild JM, Cole MD, et al. The influence of forward light scatter on the visual field indices in glaucoma. Graefes Arch Clin Exp Ophthalmol 1990;228:326-31

5 Moss ID, Wild JM. The influence of induced forward light scatter on the normal blue-on-yellow perimetric profile. Graefes Arch Clin Exp Ophthalmol 1994;232:409-14.

6 Eichenberger D, Hendrickson P, Robert $Y$, et al. Influence of ocular media on perimetric results. Doc Ophthalmol Proc Ser 1987;49:9-11.

7 Hever DK, Anderson DR, Knighton RW, et al. The influence of simulated light scattering on automated perimetric threshold measurements. Arch Ophthalmol 1988; 106:1247-51

8 Wood JM, Wild JM, Crews SJ. Induced intraocular light scatter and the sensitivity gradient of the normal visual field. Graefes Arch Clin Exp Ophthalmol 1987:225:369-73.

9 Hudson C, Khanna CJ, Miller A, et al. A method to establish the clinical relevance of a simulated cataract model. J Ophthal Photography 2003;25:80-3.

10 Chylack LT Jr, Wolfe JK, Singer DM, et al. The Lens Opacities Classification System III. The Longitudinal Study of Cataract Study Group. Arch Ophthalmol 1993;111:831-6.

11 Michelson G, Schmauss B. Two dimensional mapping of the perfusion of the retina and optic nerve head. Br J Ophthalmol 1995;79:1126-32.

12 Chauhan BC, Smith FM. Confocal scanning laser Doppler flowmetry: experiments in a model flow system. J Glaucoma 1997;6:237-45.

13 Kagemann L, Harris A, Chung H, et al. Photodetector sensitivity level and heidelberg retina flowmeter measurements in humans. Invest Ophthalmol Vis Sci 2001:42:354-7.

14 Haefliger IO, Lietz A, Griesser SM, et al. Modulation of Heidelberg Retinal Flowmeter parameter flow at the papilla of healthy subjects: effect of carbon, oxygen, high intraocular pressure, and beta-blockers. Surv Ophthalmol 1999:43(Suppl 1):S59-65.

15 Portoles M, Austin F, Nos-Barbera S, et al. Effect of poloxamer 407 on the adherence of Pseudomonas aeruginosa to corneal epithelial cells. Cornea 1995; 14:56-61.

16 Michelson G, Welzenbach J, Pal I, et al. Automatic full field analysis of perfusion images gained by scanning laser Doppler flowmetry. Br J Ophthalmol 1998;82:1294-300.

17 Kagemann L, Harris A, Chung HS, et al. Heidelberg retinal flowmetry: factors affecting blood flow measurement. Br J Ophthalmol 1998;82:131-6.

18 Jonescu-Cuypers CP, Chung HS, Kagemann L, et al. New neuroretinal rim blood flow evaluation method combining Heidelberg retina flowmetry and tomography. Br J Ophthalmol 2001;85:304-9.

19 Hosking SL, Embleton S, Kagemann L, et al. Detector sensitivity influences blood flow sampling in scanning laser Doppler flowmetry. Graefes Arch Clin Exp Ophthalmol 2001;239:407-10.

20 Tsang AC, Harris A, Kagemann L, et al. Brightness alters Heidelberg retinal flowmeter measurements in an in vitro model. Invest Ophthalmol Vis Sci 1999;40:795-9. 